\title{
Microscopía Electrónica de Nucleos Hepáticos Estimulados con Dosis Crecientes de Laser Infrarrojo
}

\author{
Electron Microscopy Hepatic Nuclei Stimulated with Increasing Doses of Infrared Laser
}

\author{
R. Cornejo*; O. Garrido** \& R. Jaramillo**
}

\begin{abstract}
CORNEJO, R.; GARRIDO, O. \& JARAMILLO, R. Microscopía eletrónica de nucleos hepáticos estimulados con dosis crecientes de laser infrarrojo. Int. J. Morphol., 33(1):222-228, 2015.

RESUMEN: Un total de 20 ratas hembras de 4 meses de vida con peso aproximado de 250 gramos fueron divididas en 4 grupos de animales rotulados como A, B, C y D. El grupo A corresponde al control y los demás grupos recibieron respectivamente estimulaciones con laser infrarrojo con dosis crecientes de 4, 8 y 16 Joules por $\mathrm{cm}^{2}$ durante 15 días consecutivos en 5 puntos del hígado. Posteriormente las ratas fueron sacrificadas y se extrajeron muestras tanto de hígado control como de los estimulados con inducciones infrarrojas para enseguida ser procesadas para microscopía electrónica de transmisión. De los hepatocitos se obtuvieron microfotografías electrónicas de transmisión con aumentos finales de $9.500 \mathrm{X}$, las cuales fueron sometidas a estudios morfométricos para determinar fracciones volumétricas de sus núcleos y estructuras nucleares. De igual manera se cuantificaron las áreas nucleares, celulares y se determinó la relación núcleocitoplasmática de los tipos celulares estudiados. Analizados los resultados entre los hepatocitos controles e irradiados se visualiza que existen notables diferencias en la totalidad de los parámetros cuantificados concluyéndose que los efectos de las estimulaciónes infrarrojas con dosis crecientes genera transformaciones en su ultraestructura y en su morfología, fundamentalmente en el aumento de los volúmenes nucleares, y celulares, los volúmenes de cromatina y de la relación-núcleo-citoplasmática situación que se traduciría en una variación funcional, representando de esta manera un efecto evidente que estas inducciones infrarrojas generan.
\end{abstract}

PALABRAS CLAVE: Láser; Morfometría; Hepatocito; Núcleos.

\section{INTRODUCCIÓN}

El uso de las emisiones derivadas del láser infrarrojo (LIR) desde la década del 80 se han constituido en una óptima terapia para el tratamiento de múltiples afecciones que incluyen procesos de cicatrización, solución a problemas musculoesqueléticos y alivio constante del dolor, logrando excelentes resultados en una amplia gama de pacientes (Millis et al., 2004)

Estos éxitos en los citados tratamientos se deben a que el láser infrarrojo de baja potencia otorga básicamente una eficaz acción cicatrizante (Castano et al., 2007), antiinflamatoria (Baybekov et al., 2008) y analgésica (Kert \& Rose, 1989).

De igual manera, las estimulaciones generadas por el LIR provocan a nivel celular, entre otros, una variada gama de mecanismos tales como: acentuada activación en la síntesis de proteínas (Omi et al., 2005), y de ciertas enzimas (Geinits et al., 2006), síntesis tanto de ATP mitocondrial (Lavi et al.,
2003) como de DNA (Loevchal \& Arenholt-Bindslev, 1994) y RNA (Karu et al., 2004) y síntesis- secreción de colágeno (Abergel et al., 1987).

Existen probadas evidencias en el sentido que el primer efecto de estimular células diversas con las emisiones generadas por el LIR es la mitocondria provocando tanto cambios de potencial en sus membranas, como en las proteínas de la cadena transportadora de electrones, en las enzimas involucradas en el Ciclo de Krebs y en la propia síntesis de ATP (Das, 2001).

En este mismo contexto, ha sido demostrado que estas modificaciones mitocondriales resultado de la estimulación con LIR desembocan finalmente en una activa comunicación con el núcleo generando una serie de eventos en condiciones normales o patológicas (Ryan \& Hoogenraad, 2007; Schroeder et al., 2007).

\footnotetext{
* Departamento de Ciencias Básicas, Facultad de Medicina, Universidad de La Frontera, Temuco, Chile.

*** Instituto de Ciencias Marinas y Limnológicas, Universidad Austral de Chile, Valdivia, Chile.
} 
En base a estos hallazgos recientemente analizados es posible argumentar que los procesos que resultan de las inducciones infrarrojas: síntesis de proteínas, de enzimas especificas, ATP, colágeno, DNA y ARN tienen como centro de generación el núcleo celular y sus componentes en términos de poseer la información genética correspondiente para codificar todas las síntesis macromoleculares que requieren de dicha información y entonces regir los procesos y mecanismos propios del ciclo vital. Del compartimento nuclear mediados por las secuencias nucleotídicas y la maquinaria enzimática replicativa y transcripcional, la información pertinente viajará al compartimento citosólico para ser traducido en macromoléculas de vital importancia para la mantención del citado ciclo vital celular (Forero \& Urcuqui, 2004).

En este mismo sentido, recientemente Zhang et al. (2003), experimentando en fibroblastos humanos demuestra que las inducciones del LIR son capaces de activar 111 genes y por consiguiente regular 10 categorías de funciones donde siete de ellas están relacionadas con el proceso de proliferación celular y las 3 restantes para regular factores de transcripción, procesos de inflamación y síntesis de citoquinas.

Todos estos hallazgos nos han estimulado para investigar intentando describir desde el punto de vista morfológico, cuantificando mediante técnicas morfométricas asociadas a microscopía electrónica, los volúmenes de núcleos y sus constituyentes pertenecientes a hepatocitos de rata estimulados con dosis crecientes de LIR

Finalmente, en investigaciones anteriores hemos determinado y publicado que hepatocitos de rata cuando estimulados con LIR con dosis correspondiente a 4,8 y $16 \mathrm{~J} /$ $\mathrm{cm} 2$ su función celular se activa notablemente con dosis correspondiente a $8 \mathrm{~J} / \mathrm{cm}^{2}$ y por el contrario dosis de $16 \mathrm{~J} / \mathrm{cm}^{2}$ la inhiben (Cornejo et al. 2011).

En la medida que se cotejen los respectivos resultados que involucran a núcleos de hepatocitos normales y los estimulados con LIR en distintas dosis se podrán establecer las variaciones en la funcionalidad que cada tipo celular posee.

\section{MATERIAL Y MÉTODO}

Un total de 20 ratas hembras de 4 meses de vida con un peso de aproximadamente 250 gramos fueron divididas en cuatro grupos de 5 animales cada uno rotulados como A, B, C, y D donde el grupo A servirá como control y los otros grupos recibieron estimulaciones infrarrojas diarias con dosis de 4,8 y 16 Joules por $\mathrm{cm}^{2}$ respectivamente en 5 puntos del hígado por espacio de 15 días consecutivos. Cumplido este tiempo las ratas fueron sacrificadas, se extrajeron muestras tanto del hígado control como de los estimulado con inducciones infrarrojas para enseguida ser procesadas con técnicas de microscopía electrónica de transmisión adicionándoles una solución de glutaraldehído $2 \%$ en tampón fosfato $0,15 \mathrm{M}, \mathrm{pH} 7,2$ manteniéndolas a temperatura ambiente por 2 horas. Luego, fueron sometidas a un lavado en solución de $6 \mathrm{~g}$ de $\mathrm{NaCl}$ y $73 \mathrm{~g}$ de sacarosa, disuelto en 1 litro de agua destilada.

La post-fijación se realizó con tetróxido de osmio $1 \%$ disuelto en la solución antes descrita durante una hora a $40^{\circ} \mathrm{C}$ y acetato de uranilo $0,5 \%$ por 18 horas. Luego de lavado el material fue deshidratado en concentraciones crecientes de acetona ( 30 a 100\%) e incluido en Araldita 6005. Se obtuvieron cortes ultrafinos de aproximadamente $70 \mathrm{~nm}$ de grosor los que fueron tratados con acetato de uranilo $2 \%$ durante 40 minutos y citrato de plomo $0,5 \%$ por 10 minutos.

Las muestras fueron finalmente estudiadas y microfotografiadas en un microscopio electrónico Phillips EM 300.

A partir de los bloques para microscopia electrónica fueron obtenidos cortes ultrafinos desde los cuales se microfotografiaron los hepatocitos con aumentos finales de hasta $9.500 \mathrm{X}$.

Para la evaluación de las fracciones volumétricas de los componentes celulares, fue sobrepuesto un retículo de puntos sobre las microfotografías electrónicas y se procedió al conteo diferencial de los puntos que incidían sobre los perfiles de los componentes, calculándose la fracción volumétrica que ellos ocupan, mediante la siguiente ecuación: (Weibel, 1969).

$$
\mathrm{Fv}=\mathrm{Pi} / \mathrm{Pt}
$$

Donde:

Fv = Fracción volumétrica del componente celular.

$\mathrm{Pi}=$ Puntos incidentes sobre el componente en estudio.

$\mathrm{Pt}=$ Puntos totales incidentes en la célula estudiada.

Para el cálculo de las áreas celulares y nucleares fue utilizado el software Sigma Scan Pro 5.0

Análisis estadístico. Todos los datos cuantitativos obtenidos fueron sometidos a la prueba de Wilcoxon mediante el paquete estadístico SPSS versión 15 para Windows (Chicago, USA) para muestras no paramétricas estableciendo diferencias con un valor de $\mathrm{Z}=2,023$ y un $\mathrm{p}=0,043$ indicando entonces que existen diferencias significativas entre ellos. 


\section{RESULTADOS}

La microscopía electrónica de transmisión utilizada en nuestro estudio muestra comparativa y eficientemente tanto la morfología celular como la núclear y sus componentes pertenecientes a hepatocitos normales y estimulados con láser infrarrojo en dosis de 4,8 y $16 \mathrm{~J} / \mathrm{cm}^{2}$, detallándose en ellos su ultraestructura evidenciados en las Figuras 1, 2, 3 y 4 respectivamente y que generan los siguientes datos morfométricos, los cuales se consignan en la Figuras 5, 6, 7, y Tablas I y II.

En el contexto de los datos morfométricos relativos a los núcleos y sus constituyentes nucleares aquí presentados muestran claramente que cuando hepatocitos de rata son estimulados con dosis de laser infrarrojo equivalente a $8 \mathrm{~J} /$ $\mathrm{cm}^{2}$ se genera una sustantiva activación de la función celular que se traduce en los siguientes hechos:

1.- El volumen nuclear expresado en fracciones volumétricas aumenta en relación directa con el incremento de la dosis de laser infrarroja utilizada hasta lograr su máximo con dosis equivalente a $8 \mathrm{~J} / \mathrm{cm}^{2}$ y que alcanza a un $15 \%$ del volumen celular.

Este dato morfométrico tiene directa relación con el área nuclear evaluada con las diferentes estimulaciones infrarrojas utilizadas, así, la mayor área equivale a 37,6 $\mu^{2}$ y se logra estimulando con dosis de $8 \mathrm{~J} / \mathrm{cm}^{2}$.

2.- En este mismo sentido, queda también claramente expuesto que el volumen nuclear de los hepatocitos estimula-

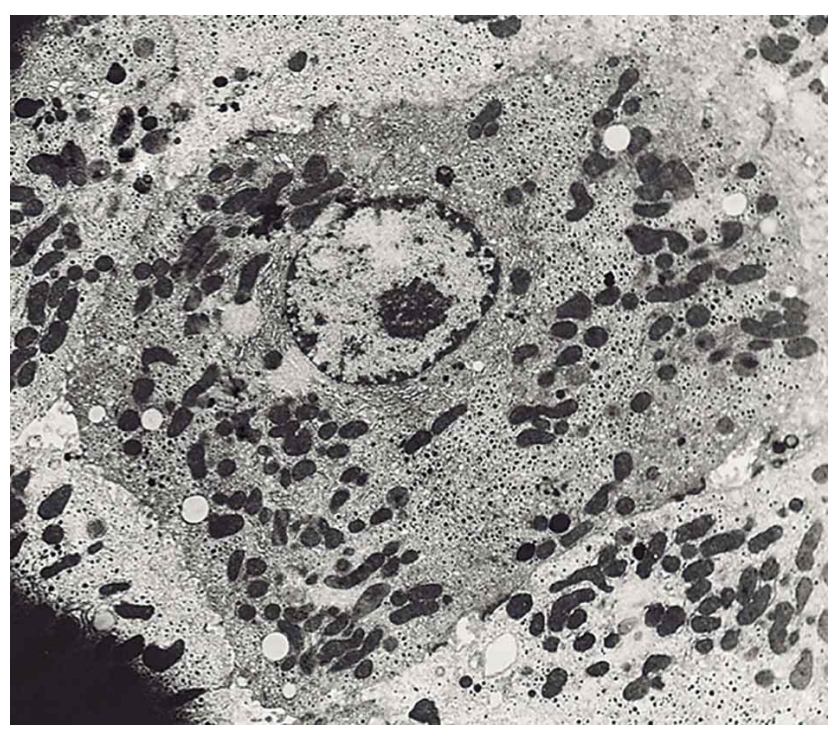

Fig. 1. Microfotografía electrónica de transmisión perteneciente a hepatocito de rata normal $9.500 \mathrm{X}$. dos con dosis superiores al óptimo como en este caso $16 \mathrm{~J} /$ $\mathrm{cm}^{2}$ genera una notable disminución de las fracciones volumétricas nucleares, disminuyendo a un $7,2 \%$ del volumen celular. Como en el caso anteriormente expuesto, el área nuclear correspondiente con esta dosis infrarroja disminuye a $22,8 \mu^{2}$.

3.- De igual manera, en lo relativo al volumen de los nucléolos cuantificados en los núcleos hepáticos es observada directa relación entre aumento de dosis utilizada en la activación versus los volúmenes nucleolares encontrados, alcanzando su mayor desarrollo también con dosis de $8 \mathrm{~J} /$ $\mathrm{cm}^{2}$ y con un $16 \%$ del volumen nuclear. Volúmenes nucleolares correspondientes a núcleos de hepatocitos estimulados con dosis mayores y menores que $8 \mathrm{~J} / \mathrm{cm}^{2}$ son evidentemente menores.

4.- En lo que respecta a los tipos de cromatina presentes en los núcleos irradiados con dosis distintas de laser infrarrojo los datos morfométricos indican claramente que con $8 \mathrm{~J} /$ $\mathrm{cm}^{2}$ la fracción volumétrica de eucromatina corresponde a la mas elevada (90\%) y por tanto la heterocromatina es, por ende la de menor volumen (10\%). Estos datos indican que este núcleo se caracteriza por una alta tasa de expresión génica.

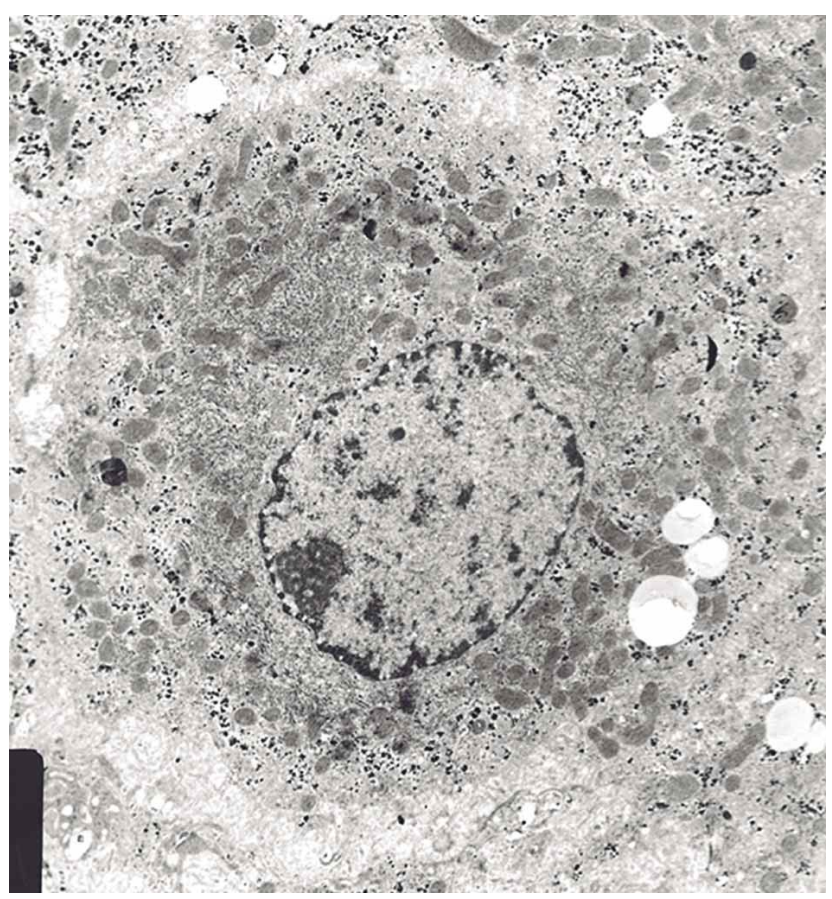

Fig. 2. Microfotografía electrónica de transmisión perteneciente a hepatocito de rata estimulado con dosis de láser infrarrojo $4 \mathrm{~J} /$ $\mathrm{cm}^{2} .9 .500 \mathrm{X}$. 


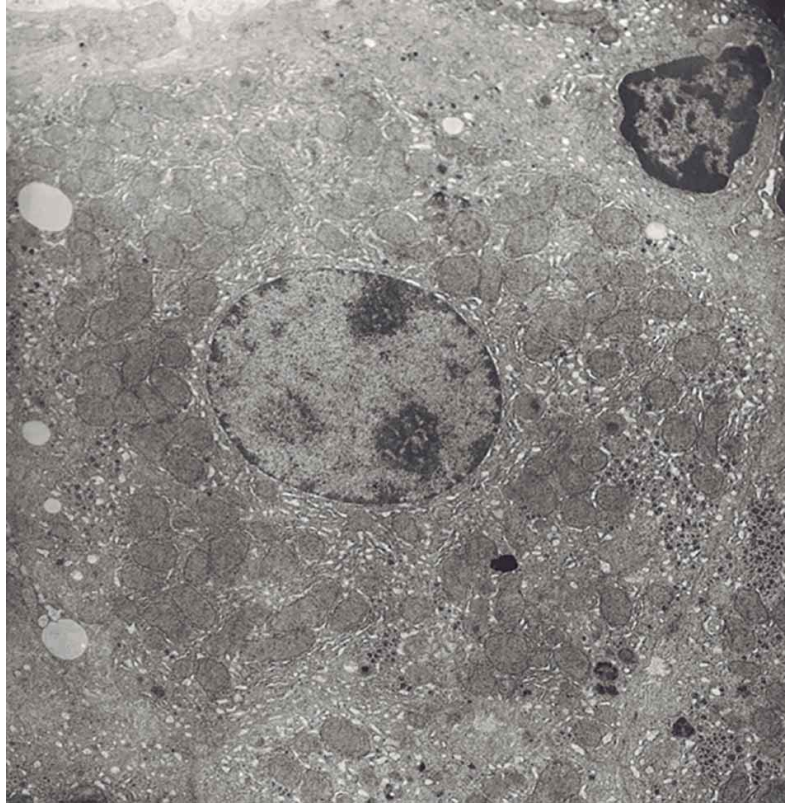

Fig. 3 Microfotografía electrónica de transmisión perteneciente a hepatocito de rata estimulado con dosis de láser infrarrojo $8 \mathrm{~J} /$ $\mathrm{cm}^{2} .9 .500 \mathrm{X}$.

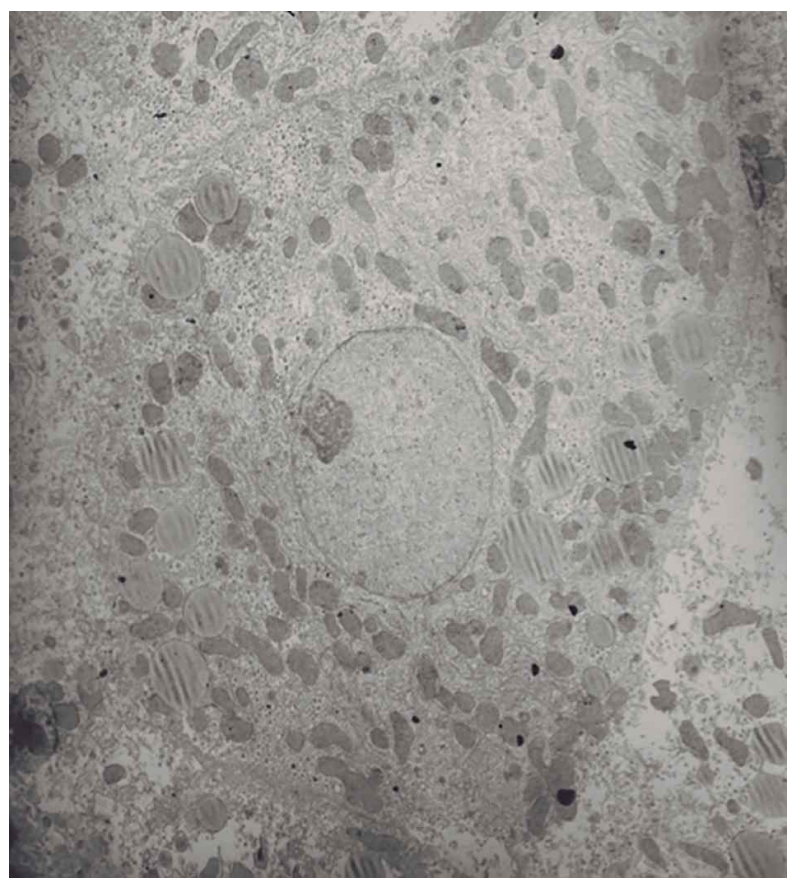

Fig. 4 Microfotografía electrónica de transmisión perteneciente a hepatocito de rata estimulado con dosis de láser infrarrojo $16 \mathrm{~J} / \mathrm{cm}^{2} .9 .500 \mathrm{X}$.

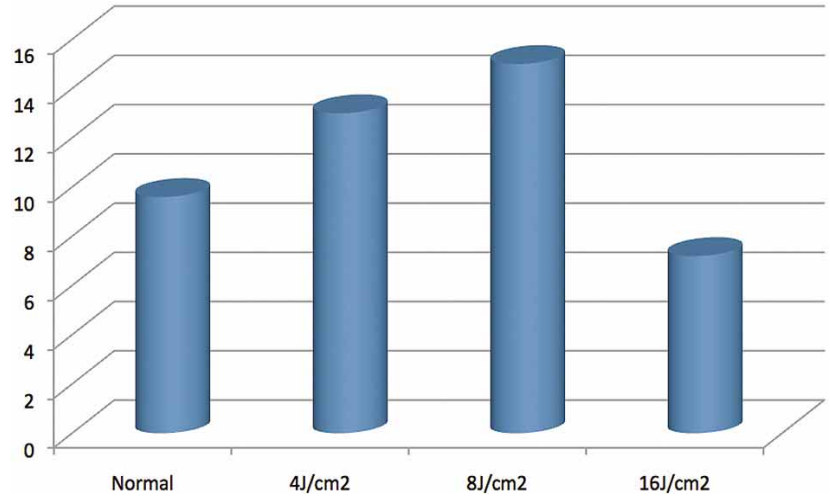

Fig. 5. Fracciones volumétricas expresadas en porcentaje correspondiente a núcleos de hepatocitos normales y estimulados con dosis de 4,8 y $16 \mathrm{~J} / \mathrm{cm}^{2}$ de laser infrarrojo.

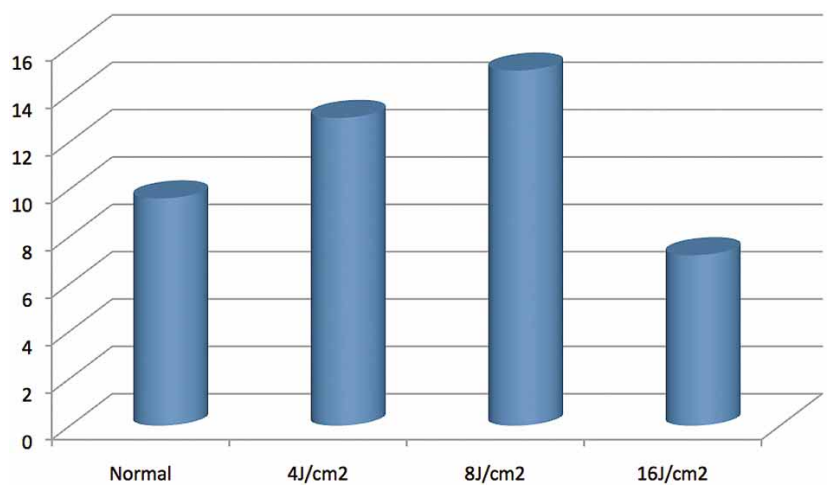

Fig 6. Fracciones volumétricas expresadas en porcentaje correspondiente a nucleólos de hepatocitos normales y estimulados con dosis de 4,8 y $16 \mathrm{~J} / \mathrm{cm}^{2}$ de laser infrarrojo.

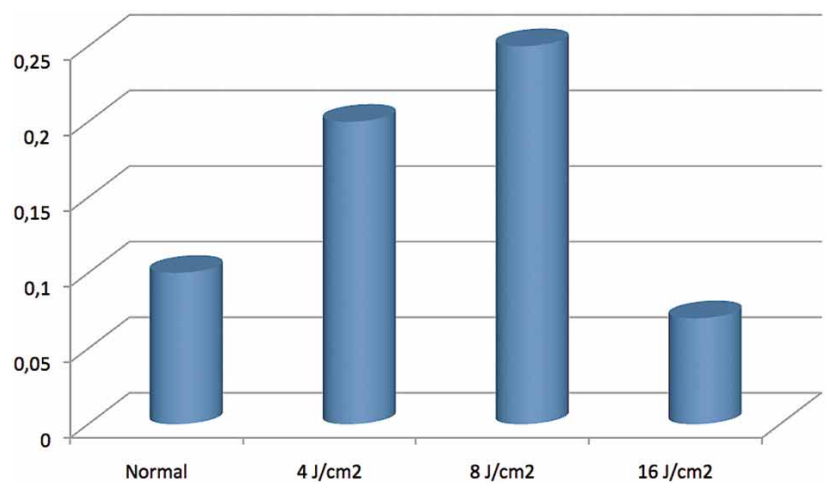

Fig. 7. Relación núcleo-citoplasmática correspondiente a hepatocitos normales y estimulados con dosis infrarrojas de 4,8 y $16 \mathrm{~J} / \mathrm{cm}^{2}$.

Tabla I. Áreas celulares y nucleares expresadas en micrones cuadrados pertenecientes a hepatocitos normales y estimulados con dosis de 4,8 y $16 \mathrm{~J} / \mathrm{cm}^{2}$ de laser infrarrojo.

\begin{tabular}{|c|c|c|c|c|}
\hline & $\begin{array}{c}\text { Hepatocito } \\
\text { Normal }\end{array}$ & $\begin{array}{c}\text { Hepatocito } \\
4 \mathrm{~J} / \mathrm{cm}^{2}\end{array}$ & $\begin{array}{c}\text { Hepatocito } \\
8 \mathrm{~J} / \mathrm{cm}^{2}\end{array}$ & $\begin{array}{c}\text { Hepatocito } \\
16 \mathrm{~J} / \mathrm{cm}^{2}\end{array}$ \\
\hline Ärea Celular & 247 & 209 & 295 & 270 \\
\hline Ärea Nuclear & 24 & 20 & 38 & 23 \\
\hline
\end{tabular}


Tabla II. Fracciones volumétricas (\%) de cromatina pertenecientes a hepatocitos normales y estimulados con dosis de 4,8 y $16 \mathrm{~J} / \mathrm{cm}^{2}$ de laser infrarrojo.

\begin{tabular}{|c|c|c|c|c|}
\hline & $\begin{array}{c}\text { Hepatocito } \\
\text { Normal }\end{array}$ & $\begin{array}{c}\text { Hepatocito } \\
4 \mathrm{~J} / \mathrm{cm}^{2}\end{array}$ & $\begin{array}{c}\text { Hepatocito } \\
8 \mathrm{~J} / \mathrm{cm}^{2}\end{array}$ & $\begin{array}{c}\text { Hepatocito } \\
16 \mathrm{~J} / \mathrm{cm}^{2}\end{array}$ \\
\hline Eucromatina & 56 & 35 & 90 & 72 \\
\hline Heterocromatina & 44 & 65 & 10 & 28 \\
\hline
\end{tabular}

Con dosis menores y mayores, los volúmenes de eucromatina disminuyen notoriamente, aumentando concomitantemente la heterocromatina.

5.- Las áreas celulares cuantificadas con las dosis infrarrojas utilizadas también indican mayor desarrollo con estimulaciones de $8 \mathrm{~J} / \mathrm{cm}^{2}$ alcanzando un área de 295 micrones cuadrados, áreas que disminuyen notoriamente con dosis de 4 y $16 \mathrm{~J} / \mathrm{cm}^{2}$

6.- La relación núcleo-citoplasmática cuantificada en estas células aumenta en directa relación con el aumento en las dosis de estimulación infrarroja, alcanzando su máximo valor también con la dosis de $8 \mathrm{~J} / \mathrm{cm}^{2}$. Esta relación decrece notoriamente cuando se aplica una dosis de $16 \mathrm{~J} / \mathrm{cm}^{2}$.

\section{DISCUSIÓN}

En el año 2010 estudiamos con microscopía óptica asociada a técnicas morfométricas el efecto que estimulaciones infrarrojas generaba tanto en las áreas de núcleos de hepatocitos como en las áreas de sus nucléolos cuando tratados con dosis correspondiente a $2,4,8$ y $16 \mathrm{~J} /$ $\mathrm{cm}^{2}$ (Cornejo et al., 2010).

Sin embargo, pareció importante estudiar y comparar los valores obtenidos en dicho estudio con microscopía óptica a otro donde a las técnicas morfométricas se asocien con microcopía electrónica de transmisión, la cual naturalmente otorgó mayor certeza y exactitud en los datos obtenidos, además que en el presente investigación cuantificamos fracciones volumétricas nucleares, nucleolares, tipos de cromatina y determinando la relación núcleo-citoplasmática y asociándolo con las áreas celulares de hepatocitos.

Efectivamente como se había planteado en las hipótesis, entre ambos estudios se encontraron grandes diferencias en los valores cuantificados de todas las estructuras celulares cuantificadas.

Existe una notable relación funcional y de estrecha dependencia entre mitocondrias y núcleo puesto que más del $90 \%$ de las proteínas mitocondriales están codificadas en el genoma nuclear y por ende, tanto la biosíntesis mitocondrial como la proliferación celular puede ser articulada en el núcleo merced a estimulaciones de factores externos tales como hormonas, temperatura e inducciones de laser infrarrojo (Ryan \& Hoogenraad).

En este contexto, Schroeder et al., trabajando en fibroblastos describen con precisión esta relación núcleomitocondrial describiendo que las emisiones infrarrojas generan un notable incremento en la función mitocondrial, incremento que se traduce en una mayor actividad en el transporte electrónico el cual incide directamente activando tanto la expresión como la transcripción génica y la posterior síntesis de metaloproteasa 1 componente de la matriz extracelular.

De igual manera, Zhang et al., irradiando fibroblastos humanos precisa que estas estimulaciones infrarrojas activan la expresión génica de 111 genes que regulan entre otras funciones la proliferación celular y la apoptosis como también genes reguladores de propiedades antioxidantes y del metabolismo energético mitocondrial.

Estos hechos se relacionan positivamente con nuestros resultados ya la relación núcleo-mitocondrial queda claramente expresada donde con dosis infrarrojas de $8 \mathrm{~J} / \mathrm{cm}^{2}$ se cuantificó $20 \%$ de volumen mitocondrial y $15 \%$ de volumen nuclear, ambos óptimos valores que apuntarían a procesos de proliferación acentuados. Mas aún, con dosis infrarrojas de $16 \mathrm{~J} / \mathrm{cm}^{2}$ se obtienen solo $14 \%$ de mitocondrias vs. $7,2 \%$ de volumen nuclear.

Por otro lado, en lo que respecta al mayor volumen nuclear cuantificado en nuestro estudio con dosis de $8 \mathrm{~J} / \mathrm{cm}^{2}$ tiene directa relación y es congruente con otros estudios en los cuales ya desde 1994 con los hallazgos de Loevschall \& Arenholt-Bindslev, se demostraba firmemente que las estimulaciones infrarrojas generaban un notable incremento en la síntesis de DNA y que se reafirma con los estudios de Ehrenhofer-Murray (2004), en el sentido que distintos factores determinan cambios en la estructura de la cromatina, especialmente en la eucromatina situación que provoca lo que ellos denominan como hiperfunción nuclear generando una evidente hiperplasia del nucléolo y la consiguiente elevada síntesis de ARNm de numerosos genes y por tanto des- 
encadenando una elevada actividad celular responsable de los mecanismos de proliferación y otros. Es preciso recalcar que con esta dosis de $8 \mathrm{~J} / \mathrm{cm} 2$ se obtiene la fracción de volumen mayor que las demás dosis ocupadas (90\%).

De igual modo, nuestros resultados con respecto a las fracciones volumétricas de los nucleólos pertenecientes a los hepatocitos estimulados con diferentes dosis de laser infrarrojo indica mas una vez que con $8 \mathrm{~J} / \mathrm{cm}^{2}$ se obtiene el mayor volumen (16\%) con la consiguiente disminución de volúmen con dosis infrarrojas mayores y menores.

Este resultado es congruente y se relacionada directamente con los resultados anteriores en el sentido que con esta dosis infrarroja se obtiene mayor volumen nuclear, mayor volumen de eucromatina, mayor volúmen mitocondrial (Cornejo et al., 2013) y además mayor volúmen nucleolar apuntando entonces a una elevada tasa de síntesis de RNAr para la biosíntesis ribosomal que, junto a los resultados presentados activarán la síntesis proteica responsable del mecanismo proliferativo (Hernandez-Verdum et al. 2010).

Observando los valores obtenidos en nuestro estudio para con la relación núcleo-citoplasmática de estos hepatocitos es posible concluir que el aumento progresivo de ella se deba a un aumento también progresivo en los volúmenes nucleares producto de las inducciones infrarrojas que presenta su máximo valor cuando las células son estimuladas con $8 \mathrm{~J} / \mathrm{cm}^{2}$, dosis con la cual se obtienen óptimos valores de cada componente celular y por tanto es tentador suponer que con ella se obtiene una mayor actividad celular, en concordancia con Junqueira \& Salles (1975).

Teniendo en cuenta entonces, los resultados nuestros y aquellos coincidentes aquí presentados es posible reafirmar nuestra hipótesis inicial en el sentido que evidenciando el desarrollo y el aumento o disminución de los volúmenes de constituyentes nucleares y de mitocondrias en hepatocitos de rata estimulados con una dosis infrarroja equivalente a 8 $\mathrm{J} / \mathrm{cm}^{2}$ se evidencia una notoria activación en la función celular, mientras que, dosis menores y mayores la disminuyen.

\section{AGRADECIMIENTOS}

Este trabajo es el resultado de las actividades de investigación realizadas en el proyecto DI-UFRO 14-0103.

CORNEJO, R.; GARRIDO, O. \& JARAMILLO, R. Electron microscopy hepatic nuclei stimulated with increasing doses of infrared laser. Int. J. Morphol., 33(1):222-228, 2015.

SUMMARY: Twenty-four four-month-old female rats weighing approximately 250 grams were divided into four groups labeled A, B, C and D. A corresponds to the normal group and the other groups received stimulation increasing doses with 4,8 and $16 \mathrm{~J} / \mathrm{cm} 2 \mathrm{of}$ infrared laser respectively for 15 consecutive days in five points of the liver. The rats were then sacrificed and samples of normal liver and liver stimulated with infrared inductions were extracted for immediate processing via transmission electron microscopy. From cell types transmission electron microphotographs were obtained at magnifications of $9500 \mathrm{X}$ these were subjected to morphometric studies to determine volumetric fractions of the nuclei and nuclear structures. Likewise, cell and nuclear areas and nuclear-citoplasmatic relation were quantified. Analysis of the results between normal and radiated hepatocytes revealed notable differences in all the cell components quantified. It is concluded that the effects of increasing infrared stimulation doses brings transformation in their ultrastructure and morphology, fundamentally in the considerable increase in nuclear volume, chromatin volume and the nuclear-citoplasmatic relation, which ultimately translates into a functional variation, thus representing an obvious impact produced by these infrared inductions.

KEY WORDS: Nuclei; Laser; Morphometry; Hepatocyte.

\section{REFERENCIAS BIBLIOGRÁFICAS}

Abergel, R. P.; Lyons, R. F.; Castel, J. C.; Dwyer, R. M. \& Uitto, J. Biostimulation of wound healing by lasers: experimental approaches in animal models and in fibroblast cultures. $J$. Dermatol. Surg. Oncol., 13(2):127-33, 1987.

Baybekov, I.; Mavlan-Khodjaev, R.; Erstekis, A. \& Moskvin, S. Erythrocytes in normal, pathology and laser effects. Moscow, Triada Publishing House, 2008.

Castano, A. P.; Dai, T.; Yaroslavsky, I.; Cohen, R.; Apruzzese, W. A.; Smotrich, M. H. \& Hamblin, M. R. Low-level laser therapy for zymosan-induced arthritis in rats: Importance of illumination time. Lasers Surg. Med., 39(6):543-50, 2007.

Cornejo, R.; Matamala Vargas, F.; Silva Mella, H.; Garrido Oñate, O.; Buchegger Mena, K. \& Jarpa Hidalgo, H. Morphometric Analysis of the Effect of Infrared Laser Stimulation on Hepatocyte Nuclei in Rats. Int. J. Morphol., 28(3):771-5, 2010.

Cornejo, R.; Matamala Vargas, F.; Silva Mella, H.; Garrido Oñate, O.; Abarzúa Bastías, Y. \& Balboa Ponce, P. Morphometric 
Study of Effect $16 \mathrm{~J} / \mathrm{cm}^{2}$ Infrared Laser in Rat Hepatocytes. Int. J. Morphol., 29(2):650-5, 2011.

Cornejo, R.; Matamala, F.; Silva, H.; Garrido, O.; Jaramillo, R.; Sáez, L.; Painemil, S. \& Loncoñanco, E. Infrared Laser Effect on Alcoholic Rat Hepatic Microvesicular Steatosis. Int. J. Morphol., 31(1):307-11, 2013.

Das, D. K. Redox regulation of cardiomyocyte survival and death. Antioxid. Redox Signal, 3(1):23-37, 2001.

Ehrenhofer-Murray, A. E. Chromatin dynamics at DNA replication, transcription and repair. Eur. J. Biochem., 271(12):2335-49, 2004.

Forero, J. E. \& Urcuqui, I. Principales factores en el transporte núcleo-citoplasma: papel de Rev en el proceso del transporte de transcritos de VIH-1. Colomb. Med., 35(4):214-23, 2004.

Geinits. A.; Moskvin, S. \& Asisov, G. Intravenous laser blood irradiation. Moscow, Triada Publushing House, 2006.

Hernandez-Verdun, D.; Roussel, P.; Thiry, M.; Sirri, V. \& Lafontaine D. L. The nucleolus: structure/function relationship in RNA metabolism. Wiley Interdiscip. Rev. RNA, 1(3):415-31, 2010.

Junqueira, L. \& Salles, M. Ultra-estrutura e funçao celular. Río de Janeiro, Guanabara Koogan, 1975.

Karu, T. I.; Pyatibrat, L. V. \& Kalendo, G. S. Photobiological modulation of cell attachment via cytochrome c oxidase. Photochem. Photobiol. Sci., 3(2):211-6, 2004.

Kert, J. \& Rose, L. Clinical laser therapy. Low level laser therapy. Veksoe, Scandinavian Medical laser Technology, 1989.

Lavi, R.; Shainberg, A.; Friedmann, H.; Shneyvays, V.; Rickover, O.; Eichler, M.; Kaplan, D. \& Lubart, R. Low energy visible light induces reactive oxygen species generation and stimulates an increase of intracellular calcium concentration in cardiac cells. J. Biol. Chem., 278(42):40917-22, 2003.

Loevschall, H. \& Arenholt-Bindslev, D. Effect of low level diode laser irradiation of human oral mucosa fibroblasts in vitro. Lasers Surg. Med., 14(4):347-54, 1994.

Millis, D. Introduction to Physical Therapy. In: Millis D.; Levine, D. \& Taylor, R. (Eds.). Canine Rehabilitation and Physical Therapy. St. Louis, Saunders, 2004. pp.5-20.

Omi, T.; Kawana, S.; Sato, S.; Takezaki, S.; Honda, M.; Igarashi, T.; Hankins, R. W.; Bjerring, P. \& Thestrup-Pedersen, K. Cutaneous immunological activation elicited by a lowfluence pulsed dye laser. Br. J. Dermatol., 153(Suppl. 2):57$62,2005$.
Ryan, M. T. \& Hoogenraad, N. J. Mitochondrial-nuclear communications. Annu. Rev. Biochem., 76:701-22, 2007.

Schroeder, P.; Pohl, C.; Calles, C.; Marks, C.; Wild, S. \& Krutmann, J. Cellular response to infrared radiation involves retrograde mitochondrial signaling. Free Radic. Biol. Med., 43(1):12835, 2007.

Weibel, E. R. Stereological principles for morphometry in electron microscopic cytology. Int. Rev. Cytol., 26:235-302, 1969.

Zhang, Y.; Song, S.; Fong, C. C.; Tsang, C. H.; Yang, Z. \& Yang, M. cDNA microarray analysis of gene expression profiles in human fibroblast cells irradiated with red light. J. Invest. Dermatol., 120(5):849-57, 2003.

Dirección para Correspondencia:

Dr. Ricardo Cornejo

Facultad de Medicina

Universidad de La Frontera

Casilla 54-D

Temuco

CHILE

Email: rene.cornejo@ufrontera.cl

Recibido : 01-10-2014

Aceptado: 07-01-2015 